blood samples taken before (sometimes by many years) their diagnosis of prostate cancer. These studies included data on serum endogenous sex-hormone levels from a total of 3,886 men with incident prostate cancer and 6,438 control individuals.

There were no statistically significant associations between levels of any of the sex hormones (including total testosterone, calculated free testosterone, dihydrotestosterone, androstenediol glucuronide, androstenedione, dehydroepiandrosterone sulfate, estradiol and calculated free estradiol) and risk of prostate cancer; adjustment for confounding variables made no difference to these findings. The highest quintile of serum sex-hormone-binding globulin level was, however, associated with significantly decreased prostate cancer risk compared with the lowest quintile (relative risk $0.86,95 \% \mathrm{Cl} 0.75-0.98$ ).

The authors conclude that prediagnosis serum levels of endogenous sex hormones are not associated with the risk of developing prostate cancer.

Original article Endogenous Hormones and Prostate Cancer Collaborative Group (2008) Endogenous sex hormones and prostate cancer: a collaborative analysis of 18 prospective studies. J Natl Cancer Inst 100: 170-183

\section{Reduced likelihood of RCC in patients taking statins}

Statins have been reported to have anticancer activity in various urological cancers, including bladder and prostate cancer. Khurana and colleagues have used data from the US South Central Veterans Integrated Service Network (a veterans' health care system that covers eight US states) to investigate the effect of statin use on the incidence of renal cell carcinoma (RCC). In total, 483,733 individuals who visited this health care system from October 1998 to June 2004 were included in the retrospective case-control study.

A primary diagnosis of RCC was identified in 1,446 patients (0.30\%). Statin use was recorded in 164,441 patients; 432 (0.26\%) of these individuals subsequently developed RCC, compared with 1,014 (0.32\%) of those who were not taking statins. After adjustment for age, ethnicity, sex, BMI and smoking status, patients taking statins were only half as likely as those who were not on these agents to develop RCC (odds ratio 0.52, 95\% Cl 0.45-0.60).
This reduction in risk was seen irrespective of age, BMI or smoking status.

The authors conclude that statin use was independently associated with a reduced likelihood of developing RCC. As with other types of cancer, randomized controlled trials will be necessary to confirm the effect of statins in the prevention and treatment of RCC.

Original article Khurana V et al. (2007) Statins might reduce risk of renal cell carcinoma in humans: case-control study of 500,000 veterans. Urology 71: 118-122

\section{Newly identified loci associated with prostate cancer risk}

Three papers published in Nature Genetics have investigated the genetic basis of prostate cancer, and identified new loci associated with risk for the disease. The identification of such loci offers the potential for improved risk stratification and novel therapeutic targets.

In the first paper, Eeles et al. report a twostage, genome-wide association study (GWAS) conducted in men from the UK and Australia. The authors confirmed associations between the previously identified loci 8q24 and 17q and prostate cancer risk, and identified a further seven loci on chromosomes $3,6,7,10,11,19$ and $X$ that had significant associations with the disease. The most promising candidate genes included MSMB, KLK3, LMTK2, NUDT10 and NUDT11.

Thomas et al. report the results of a different two-stage GWAS conducted in US men of European ancestry. Associations between prostate cancer and 8q24 and 17q were confirmed, and further significantly associated loci were identified on chromosomes 7, 10 and 11. Candidate genes included MSMB, CTBP2 and JAZF1.

The third study, by Gudmundsson et al., investigated two single-nucleotide polymorphisms (identified in an initial Icelandic GWAS) in seven European and US cohorts. Both polymorphisms, on chromosomes Xp11.22 and 2p15, were significantly associated with prostate cancer risk. Candidate genes included EHBP1, GSPT2, MAGED1, NUDT10 and NUDT11.

Original articles Eeles RA et al. (2008) Multiple newly identified loci associated with prostate cancer susceptibility. Nat Genet 40: 316-321

Thomas Get al. (2008) Multiple loci identified in a genomewide association study of prostate cancer. Nat Genet 40:310-315 Gudmundsson J et al. (2008) Common sequence variants on 2 p15 and Xp11.22 confer susceptibility to prostate cancer.

Nat Genet 40: 281-283 